\title{
Structure and Dynamics in Aqueous Solutions of Amphiphilic Sodium Maleate-Containing Alternating Copolymers
}

\author{
E. Di Cola, ${ }^{\dagger}$ N. Plucktaveesak, ${ }^{\ddagger}$ T. A. Waigh,,$^{* \dagger}$ R. H. Colby, J . S. Tan, ${ }^{\S}$ \\ W. Pyckhout-Hintzen, ${ }^{\perp}$ and R. K. Heenan ${ }^{\#}$
}

Polymers and Complex Fluids, School of Physics and Astronomy, University of Leeds, Leeds, LS2 9J T, UK; Department of Materials Science and Engineering, Pennsylvania State University, University Park, Pennsylvania 16802; Imaging Materials and Media, Research and Development, Eastman Kodak Company, Rochester, New York 14650-2116;

Institut für Festkörperforschung, Forschungszentrum J ülich, D-52425 J ülich, Germany; and

Rutherford Appleton Laboratory, Chilton, Didcot, Oxon OX11 0QX, UK

Received April 16, 2004; Revised Manuscript Received August 18, 2004

\begin{abstract}
The structure of aqueous solutions of sodium maleate copolymers with comonomers of variable hydrophobicity are examined using a combination of small-angle neutron scattering, small-angle $X$-ray scattering, and rheometry. Semidilute solutions of the copolymers made with mildly hydrophobic comonomers exhi bit scattering and rheology that are typical of flexi ble polyelectrolytes, with a correlation length scaling with polymer concentration as $\mathrm{c}^{-\alpha}$ with $\alpha=1 / 2$ and specific viscosity scaling as $\mathrm{c}^{1 / 2} / \mathrm{c}^{3 / 2}$ when unentangled/entangled. In contrast, copolymers with more than eight carbons in their hydrocarbon comonomer form micelles (globules) at low concentrations and molecular weights. For these more hydrophobic copol ymers, the correlation length scales as $\mathrm{c}^{-\alpha}$ with $\alpha<1 / 3$ and the viscosity remains small until a relatively high concentration at which the globules start to overlap. Once these more hydrophobic copolymers overlap, the micelles open up and allow intermolecular associations that cause the viscosity to increase rapidly with concentration, typical of associating polymer solutions.
\end{abstract}

\section{Introduction}

The theoretical ${ }^{1,2}$ and experimental ${ }^{3-7}$ behavior of hydrophobically modified polyelectrolytes has recently experienced some important developments. The thermodynamics of dilute solutions has been calculated, and predictions for the morphology have been made. ${ }^{1}$ Progress in the field is driven by the diverse range of industrial applications of hydrogel viscosity modifiers for use in photographic products, oil reclamation, drug delivery, or personal care products. ${ }^{3}$

There are three closely related fiel ds which the reader should differentiate between hydrophobic polyelectroIytes, ${ }^{8}$ hydrophobically modified polyelectrolytes (HMPEs), ${ }^{1}$ and polysoaps, ${ }^{9}$ classified in order of the degree of increasing chemical complexity. Hydrophobic polyel ectrolytes are charged homopolymers in sol vents that are considered poor for the conformations of the chain backbone. The competition between el ectrostatic forces and the hydrophobicity of the chain make aqueous solutions of this class of polymers exhibit a range of novel behavior. ${ }^{10}$ Recent progress has been made with respect to the Rayleigh charge instability in these systems due to the interplay between the el ectrostatics and the surface tension of the globular morphologies. ${ }^{11,12}$ $X$-ray and neutron evidence has been found for three separate synthetic polyelectrolyte systems, indicating a change in the scaling of the semi dilute mesh size due to the effects of hydrophobicity. ${ }^{13-17}$ Atomic force microscopy also has found evidence for a globular instabil-

\footnotetext{
† University of Leeds.

¥ Pennsylvania State University.

$\S$ Eastman Kodak Company.

$\perp$ Institut für Festkörperforschung.

\# Rutherford Appleton Laboratory.

* To whom correspondence should be addressed. E-mail t.a. waigh@eeds.ac.uk.
}

ity on synthetic flexible polyelectrolytes through direct imaging. ${ }^{18} \mathrm{For}$ semiflexible polyelectrolytes in poor solvents analogous toroidal necklace structures have also been found. 19,20

HMPEs are charged copolymers, containing hydrophobic comonomers that act as stickers, forming intramolecular associations in dilute solutions and viscoelastic gels at high polymer concentrations in water. Polysoaps show many similarities to HMPEs and the names are sometimes used interchangeably in the literature, the difference being the emphasis on an amphiphilic modification with polysoaps; i.e., the pendant group is amphiphilic, and the headgroup area determines the micellar aggregation number. ${ }^{9,21}$ The theory has developed separately with these materials, and the connection to fibrous protein conformations has been made. ${ }^{21}$ An example of a biological analogue is titin. 21 Titin has an immunoglobulin domain motif, which consists of globular beta barrel structures separated by flexible linker regions and thus has a polyglobular structure analogous to the predictions for HMPEs and polysoaps. ${ }^{21}$ The dynamics of hydrophilic and hydrophobic polyel ectrolytes has made some progress using dynamic scaling theory. ${ }^{22-24}$

This framework provides a common basis for the understanding of scattering, osmotic pressure, diffusion, and rheology. Models for the structure of hydrophobically modified polyelectrolytes have only been partially developed, and their dynamics has hardly been approached. ${ }^{1,2}$ Such associating polymers show numerous novel phenomena, including shear thickening rheology and the formation of hydrogels, ${ }^{4}$ indicating a large scope for designing rheological properties. ${ }^{25}$

Alternating lithium maleate copolymers with varying side chain lengths of alkene pendant group have been the subject of a previous SANS study in dilute/semidilute solution, 26,27 and similar materials are examined 
Table 1. Molecular Weight and Molecular Weight Distribution of Sodium Maleate Alternating Copolymers

\begin{tabular}{|c|c|c|c|c|c|}
\hline copolymer & molar mass $(\mathrm{g} / \mathrm{mol})$ & $M_{w} / M_{n}$ & monomer molar mass & morphology & supplier \\
\hline \multicolumn{6}{|c|}{ Poly(isobutylene-alt-maleate) } \\
\hline IBMA04 & 82000 & 2.6 & 216 & linear & Kuraray America, Inc \\
\hline IBMA10 & 196000 & 3.0 & 216 & linear & Kuraray America, Inc \\
\hline IBMA18 & 410000 & 2.4 & 216 & linear & Kuraray America, Inc \\
\hline \multicolumn{6}{|c|}{ Poly(diisobutylene-alt-maleate) } \\
\hline DIBMA & $15000^{a}$ & 1.4 & 272 & linear associating & Kodak \\
\hline \multicolumn{6}{|c|}{ Poly(styrene-alt-maleate) } \\
\hline SMA & 350000 & 4.7 & 264 & linear & Scientific Polymer Products \\
\hline \multicolumn{6}{|c|}{ Poly(n-alkene-alt-maleate) } \\
\hline $\mathrm{n}-\mathrm{C}_{8} \mathrm{MA}$ & 67500 & 3.7 & 272 & $\begin{array}{l}\text { micellar necklace/ } \\
\text { cylindrical micelle }\end{array}$ & S.C. J ohnson \& Sons \\
\hline $\mathrm{n}-\mathrm{C}_{10} \mathrm{MA}$ & 23700 & 2.0 & 300 & globular & Kodak \\
\hline $\mathrm{n}-\mathrm{C}_{12} \mathrm{MA}$ & 27100 & 2.1 & 328 & globular & Kodak \\
\hline $\mathrm{n}-\mathrm{C}_{14} \mathrm{MA}$ & 31700 & 2.5 & 356 & globular & Kodak \\
\hline
\end{tabular}

a DIBMA aggregates in solution, making the reported molecular weight unreliable.

in the current investigation. Maleic anhydrides represent a well-characterized polyelectrolyte system with respect to both the polymer stoichiometry and molecular weight. A change of morphology is thought to occur for these alternating copolymers, once the number of carbons in the alkene comonomer is increased to $8 / 10$ carbons. Linear extended polymer conformations occur with shorter pendant groups, and globular morphologies are seen with longer pendant groups. ${ }^{26,27}$ From fits of form factors to SANS data, the intermediate hydrophobically modified polyelectrolytes are thought to adopt ellipsoidal morphologies ${ }^{27}$ with the hydrophobic moieties placed in the core of the micelles (n- $C_{8}, n-C_{10}$ alkene comonomers). More hydrophobic polymers are thought to adopt cylindrical morphologies. These conclusions were drawn from the analysis of powder averaged SANS data assuming no interaction between the geometrical units. ${ }^{27}$ From a more robust scaling analysis of SANS/ SAXS and rheology data we have altered this categorization.

An extension in the present work is to examine these polymers in the framework of scal ing theories and with a combination of complementary techniques: smallangle X-ray scattering, small-angle neutron scattering, and solution state rheology. Using a combination of these methods, a more detailed picture of the structure and dynamics of the aqueous solutions is available. Dynamic light scattering suggests that sodium maleate copolymers with relatively hydrophilic comonomers (with short pendant group lengths) are standard flexible polyel ectrolytes. ${ }^{28}$ Dynamic light scattering on solutions of copolymers with longer hydrophobic pendant groups in combination with particle tracking microrheology shall be presented separately. ${ }^{29}$

In the present work we differentiate between hydrophobic polyelectrolyte and hydrophobically modified polyel ectrolyte behavior as a function of the comonomer hydrophobicity in a series of sodium maleate alternating copolymers. The sodium mal eate copolymers with mildly hydrophobic comonomers are standard flexible hydrophobic polyelectrolytes, ${ }^{22}$ whereas more hydrophobic hydrocarbon comonomers (with 10 or more carbons) exhibit the associating properties of HMPEs. ${ }^{27,4}$ The copolymer made from n-octene forms an intriguing intermediate case between HMPEs and hydrophobic polyelectrolytes. The properties of the aqueous solutions of these copolymers are categorized in terms of their dynamics and static structure.

\section{Experimental Section}

Samples. The sodium maleate copolymers investigated in this work were kindly provided by a number of sources as shown in Table 1. All of the polymers studied are alternating copolymers, and their local structure was checked with ${ }^{13} \mathrm{C}$ NMR and titration. ${ }^{30}$ The name HMPEs does not now necessitate that they are achieved by postpolymerization chemistry; it is considered to be a more general classification. ${ }^{1}$ The molecular weights and the molecular weight distributions are also indicated. The copolymers included isobutylene (IBMA), di isobutylene (DIBMA), styrene (SMA), and 1-alkenes with $m$ $=8,10,12,14$ carbons $\left(n-C_{m} M A\right)$. The mal eic anhydride rings were hydrolyzed with excess $\mathrm{NaOH}$ at $50^{\circ} \mathrm{C}$ to ensure that the maleic anhydride groups had been changed to sodium maleate. Subsequently, the samples were dialyzed against dei onized distilled water (dialysate conductivity $\leq 2 \mu \mathrm{S} / \mathrm{cm}$ ). Samples were freeze-dried and vacuum oven-dried at $40^{\circ} \mathrm{C}$. When dissolved in water with no added salt, the $\mathrm{pH}$ was $\approx 8$ for these copolymer solutions. The composition, dissociation behavior, aggregation state, and size of these copolymers in dilute aqueous solutions were the subject of previous work. ${ }^{30,31}$ No phase separation was seen for the materials up to high pol ymer concentrations (30\% w/w) unless salt was added.

Small-Angle Neutron Scattering (SANS). (a) LOQ. SANS data were measured on the LOQ small-angle diffractometer at the ISIS Spallation Neutron Source (Rutherford Appleton Laboratory, Didcot, U.K.).32,33 This is a fixedgeometry "white beam" time-of-flight instrument, which utilizes neutrons with wavelengths, $\lambda$, between 2 and $10 \AA$ to provide a simultaneous q range of $0.01-0.27 \AA^{-1}(24-630 \AA$ in real space). The momentum transfer $q=(4 \pi / \lambda) \sin \vartheta / 2$, where $\vartheta$ is the angle at which neutrons scatter. Each raw scattering data set was corrected for the sample transmission and background scattering and converted to scattering crosssection data $(\partial \Sigma / \partial \Omega$ vs q) using the instrument-specific software. These data were placed on an absolute scale $\left(\mathrm{cm}^{-1}\right)$ using the scattering from a standard sample (a solid blend of hydrogenous and perdeuterated polystyrene) in accordance with established procedures. ${ }^{34}$

Measurements were taken over the range $1-30 \% \mathrm{w} / \mathrm{w}$ using $1 \mathrm{~mL}$ fused silica cells with path lengths of 1 and $2 \mathrm{~mm}$. Samples prepared in $\mathrm{D}_{2} \mathrm{O}$ were left to equilibrate for $48 \mathrm{~h}$. The $\mathrm{D}_{2} \mathrm{O}$ solvent increased the available contrast, reduced the incoherent background, and would only be expected to have a small effect on the conformation of the neutralized polyelectrolytes.

(b) KWS2. SANS measurements were completed on KWS2 at the research reactor FRJ 2 (Forschungszentrum J ulich $\mathrm{GmbH}$ ), a classical pinhole instrument with wavelength distribution $\Delta \lambda / \lambda=10 \%$. The $\mathrm{q}$ range is from $10^{-3}$ to $0.2 \AA^{-1}$ (real space 6000 to $30 \AA$ ). KWS2 uses a 2D multiwire detector filled with ${ }^{3} \mathrm{He}$ that can be moved continuously from a distance of $1.25-20 \mathrm{~m}$ from the samples, so a combination of different camera lengths was used in order to obtain the low q data (8, 


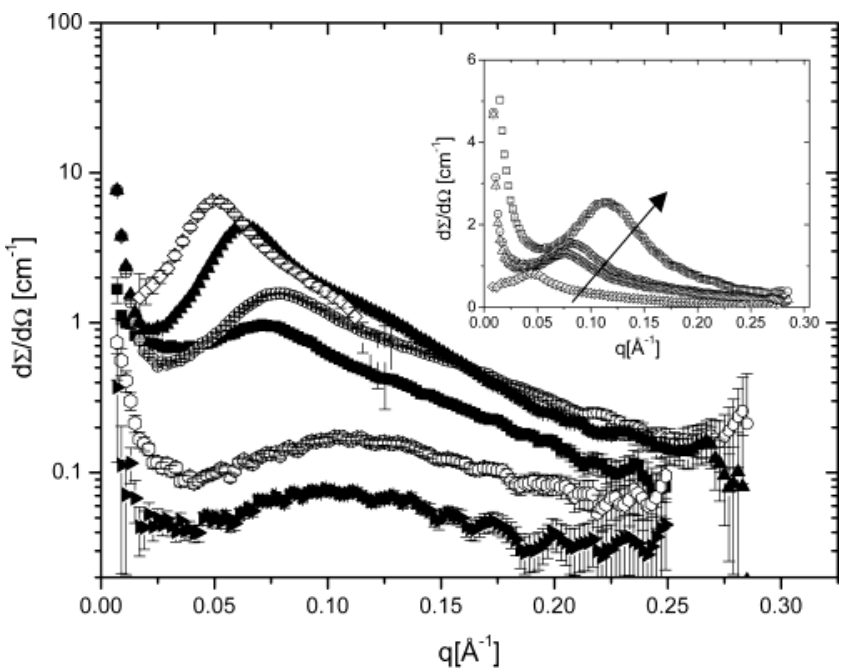

Figure 1. SANS intensity profiles for copolymers at 0.22 mol/L concentration: $n-C_{8} M A$ (filled squares), $n-C_{10} M A$ (open circles), n- $\mathrm{C}_{12} \mathrm{MA}$ (filled triangles), $\mathrm{n}-\mathrm{C}_{14} \mathrm{MA}$ (open diamonds), SMA (filled arrows), and DIBMA (open hexagons). Inset: the concentration dependence of SANS intensity profiles for the $\mathrm{n}-\mathrm{C}_{8} \mathrm{MA}$ copolymer at concentrations $0.73 \mathrm{~mol} / \mathrm{L}$ (open squares), $0.38 \mathrm{~mol} / \mathrm{L}$ (open circles), $0.22 \mathrm{~mol} / \mathrm{L}$ (open triangles), and 0.055 $\mathrm{mol} / \mathrm{L}$ (open diamonds). Arrow indicates increasing polymer concentration.

2, and $1.4 \mathrm{~m}$ ). The raw two-dimensional data were corrected for the empty cell scattering and $\mathrm{D}_{2} \mathrm{O}$ background. The detector sensitivity corrections were done pixel wise in $2 \mathrm{D}$, and the calibration intensity was made on the incoherent flat level of a Lupolene standard $\left(\mathrm{d} \Sigma / \mathrm{d} \Omega=1.78155 \mathrm{~cm}^{-1}\right)$ previously calibrated to vanadium.

Small-Angle X-ray Scattering (SAXS). SAXS experiments were conducted at the Daresbury synchrotron on the NCD beamlines designated 8.2, 2.1, and 16.1 operating at fixed wavelength $(\lambda)$ of $1.54 \AA .{ }^{35,36}$ Reciprocal space was calibrated with a rat tail collagen standard and silver behenate. A combination of different camera lengths 1 and $3.5 \mathrm{~m}$ on 8.2, $6.25 \mathrm{~m}$ on 2.1 , and $1.5 \mathrm{~m}$ on 16.1 were used, providing access to length scales in the range 10-2000 $\AA$.

Furthermore, an in-house X-ray facility was used to study the high momentum transfer structure of the samples and confirm the synchrotron results. The system operated with a high star multiwire 2D detector and $\mathrm{Cu} \mathrm{K} \alpha$ target with a camera length of $0.42 \mathrm{~m}$, providing a q range between 0.02 and $0.75 \AA^{-1}(8-300 \AA)$.

Rheology. Rheology measurements were performed with a Contraves low shear 30 viscometer, a strain-controlled rheometer equipped with a concentric cylinder geometry with a $12.0 \mathrm{~mm}$ diameter measuring cup and $11.1 \mathrm{~mm}$ diameter bob, $8 \mathrm{~mm}$ long. Temperature was controlled to $25.0 \pm 0.2^{\circ} \mathrm{C}$ with a water circulator. Steady-state measurements were performed in the shear rate range of $0.02 \mathrm{~s}^{-1} \leq \dot{\gamma} \leq 100 \mathrm{~s}^{-1}$.

\section{Results}

SANS and SAXS. Neutron scattering profiles from a range of sodium maleate copolymers at the same concentration are shown in Figure 1. As an example, SANS curves from $n-C_{8}$ MA polyelectrolyte solutions at four concentrations are shown in the inset. The scattering cross section $(\mathrm{d} \Sigma / \mathrm{d} \Omega)$ for neutrons is defined as

$$
\frac{\mathrm{d} \Sigma}{\mathrm{d} \Omega}=m \mathrm{~V}^{2}(\Delta \rho)^{2} \mathrm{P}(\mathrm{q}) \mathrm{S}(\mathrm{q})
$$

where $m$ is the number concentration of scattering centers, $\mathrm{V}$ is the volume of one scattering center, and $\Delta \rho=\left(\rho-\rho_{\mathrm{m}}\right)$ is the contrast factor between the polymer and the matrix solvent, evaluated on the basis of

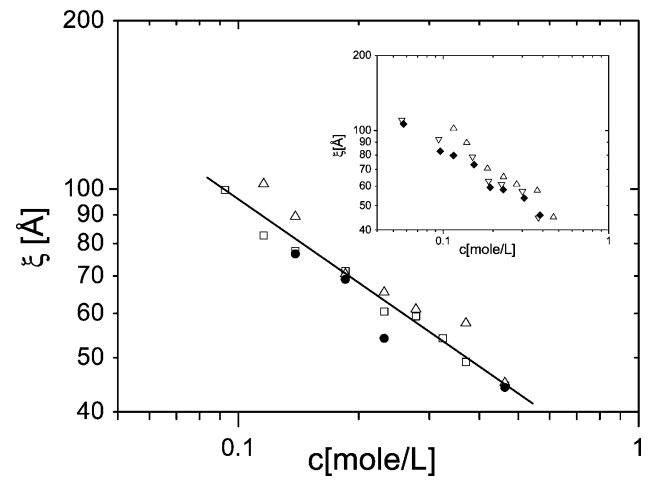

Figure 2. Mesh size $\left(\xi=2 \pi / \mathrm{q}_{\max }\right)$ from SAXS data as a function of concentration for the poly(isobutylene-alt-sodium maleate) copolymers of three different molecular weights: $M_{w}$ $=82000 \mathrm{~g} / \mathrm{mol}$ (open squares), $M_{w}=196000 \mathrm{~g} / \mathrm{mol}$ (open triangles), $M_{w}=410000 \mathrm{~g} / \mathrm{mol}$ (filled circles). The line is a least-squares fit to a power law (eq 3 with $\alpha=0.47$ ). Inset: the mesh size from SAXS data as a function of polymer concentration for copolymers with different weakly hydrophobic comonomers: SMA (open down triangles), DIBMA (filled diamonds), and IBMA04 (open squares), with exponent values listed in Table 2.

tabulated values of the coherent scattering lengths $b_{i}$; $P(q)$ and $S(q)$ indicate the form and the structure factor, respectively. ${ }^{37}$ The scattering length density $(\rho)$ of a molecule with $\mathrm{i}$ atoms can be calculated from the following expression: ${ }^{37}$

$$
\rho=m \sum_{\mathrm{i}} \mathrm{b}_{\mathrm{i}}=\delta \mathrm{N}_{\mathrm{Av}} / \mathrm{M} \sum_{\mathrm{i}} \mathrm{b}_{\mathrm{i}}
$$

where $\delta$ is the bulk density of the molecule, $\mathrm{M}$ is the molecular weight, and $\mathrm{N}_{\mathrm{Av}}$ is Avogadro's number.

Over the concentration range from $1 \%$ to $30 \% \mathrm{w} / \mathrm{w}$ there is a single peak in the small-angle neutron scattering profiles shifting toward higher angles with increasing polyelectrolyte concentration for all the copolymers investigated (Figure 1 inset). The wavevector of the local maximum in intensity $\left(q_{\max }\right)$ is related to the average mesh size $\xi$ of the solution 22 as $\xi=2 \pi /$ $q_{\max }$. As expected, the mesh size decreases as the copolymer concentration is increased. Comparison of the SANS data from the complete series of polyel ectrolytes at a single monomer concentration $(0.2 \mathrm{~mol} / \mathrm{L})$ clearly shows a sharp change in the scattering function between $n-C_{m} M A(m \geq 8)$ and the less hydrophobic polyelectrolytes (Figure 1). There is an order of magnitude increase in the scattered intensity, with the small-angle peak becoming sharper and moving to larger length scales.

In Figure 2 the scaling of $\xi$ as a function of the concentration, from SAXS data, is shown for the poly(isobutylene-alt-sodium mal eate) (IBMA) copolymers for three different molecular weights. We use SAXS data in this case because the small-angle peaks were better resolved than in their neutron counterparts, although valuable information is available using the two techniques with respect to the different contrast mechanisms. The data follow the prediction for standard flexible polyelectrolytes since the mesh size scales as

$$
\xi \sim \mathrm{c}^{-\alpha}
$$

with $\alpha=0.47 \pm 0.05$ for IBM A and $\alpha=1 / 2$ predicted by the scaling theory.

Similar behavior was observed for the copolymers with styrene and diisobutylene comonomers, and a 


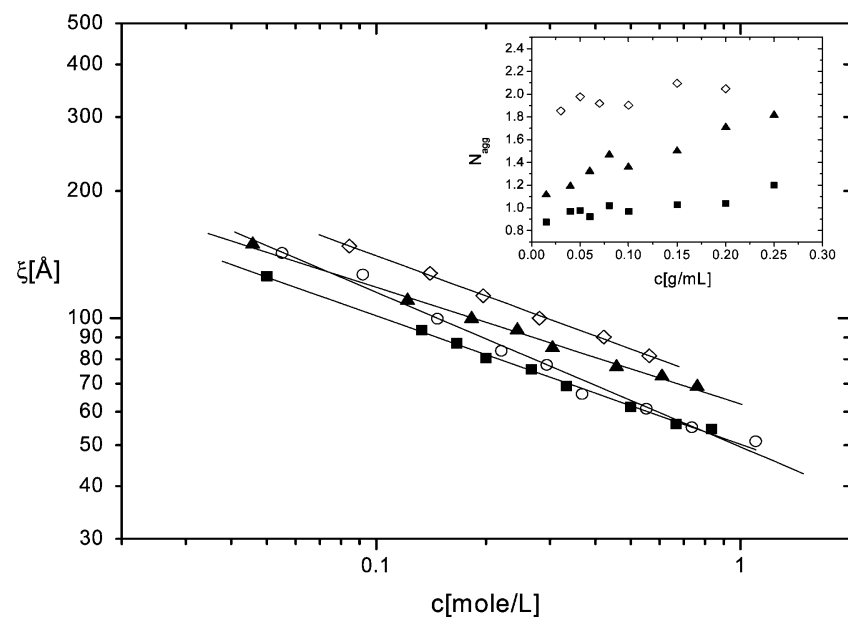

Figure 3. Mesh size from SANS data as a function of polymer concentration for poly(1-alkene-alt-sodium maleate) copolymers with different degrees of hydrophobicity: $n-\mathrm{C}_{8} \mathrm{MA}$ (open circles), $\mathrm{n}-\mathrm{C}_{10} \mathrm{MA}$ (filled squares), $\mathrm{n}-\mathrm{C}_{12} \mathrm{MA}$ (filled triangles), $\mathrm{n}-\mathrm{C}_{14} \mathrm{MA}$ (open diamonds). Solid lines are least-squares fits to power laws, with exponent values listed in Table 2. Inset: the variation of aggregation number with concentration calculated with eq 6.

master pl ot of the mesh size $\xi$ from SAXS as a function of the concentration is shown in the inset to Figure 2.

A similar analysis of the neutron intensity scattering profiles was carried for the poly(1-alkene-alt-sodium maleate) copolymers as a function of the degree of hydrophobicity, controlled by varying the number of carbons in the al kene comonomer from 8 to 14 carbons. The scaling of the structure factor peak with concentration reduces from the standard $\alpha=1 / 2$ to $\alpha<1 / 3$, as shown in Figure 3. Differences in values of $\alpha$ from SAXS and SANS are due to different contrast mechanisms. SAXS probes the counterion distribution ( $\mathrm{Na}^{+}$ions), and SANS is more sensitive to the hydrogens in the polymer backbone and side chains.

SAXS data from the hydrophobically modified polyelectrolyte $n-C_{8} M A$ at different concentrations shows similar behavior to that seen in SANS in Figure 1. Again, a peak is seen in the scattering profiles, which moves to higher $\mathrm{q}$ as the polymer concentration is increased. The scaling of the mesh size was studied as a function of concentration for various HMPEs, and the results are plotted in Figure 4. It is interesting to note for the $n-C_{8} M A$ copolymer that the scaling exponent collapses to a value of $\alpha=0.15$ as the concentration increases above $0.37 \mathrm{~mol} / \mathrm{L}$ (see the inset of Figure 4). In Table 2 a summary of the scaling exponents from SAXS and SANS measurements of the sodi um mal eate copolymers is shown. A second oscillation in the structure factor was observed in the high q range for the high hydrophobicity $n-C_{m} M A$ samples ( $m \geq 8$, Figure 5 ); however, for $\mathrm{n}-\mathrm{C}_{8} \mathrm{MA}$ the second peak was observed only for concentrations above $0.29 \mathrm{~mol} / \mathrm{L}$.

Rheology. Solution viscosity as a function of shear rate was measured to determine the zero shear rate viscosity $\eta_{0}$. The specific viscosity $\eta_{\mathrm{sp}}\left(\eta_{\mathrm{sp}}=\left(\eta_{0}-\eta_{\mathrm{s}}\right) / \eta_{\mathrm{s}}\right.$, where $\eta_{\mathrm{s}}$ is the solvent viscosity) was then calculated and plotted as a function of the polymer concentration. There is a crossover from $\eta_{\mathrm{sp}} \sim \mathrm{c}^{1 / 2}$ (unentangled) at low concentrations to $\eta_{\mathrm{sp}} \sim \mathrm{c}^{3 / 2}$ (entangled) at high concentrations for the samples of low hydrophobicity. In Figure 6 the concentration dependence of the specific viscosity is shown for poly(isobutylene-alt-sodium mal eate) (IBMA)

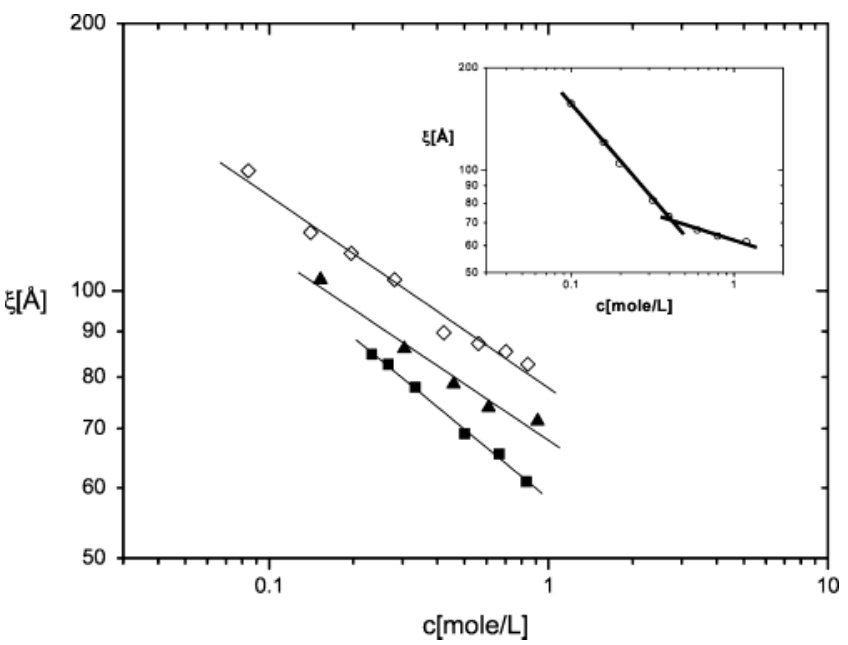

Figure 4. Mesh size from SAXS as a function of polymer concentration for poly(1-alkene-alt-sodium maleate) copolymer HMPEs: $n-C_{8} M A$ (inset: open circles), $n-C_{10} M A$ (filled squares), $\mathrm{n}-\mathrm{C}_{12} \mathrm{MA}$ (filled triangles), $\mathrm{n}-\mathrm{C}_{14} \mathrm{MA}$ (open diamonds). Thelines are least-squares fits to power laws, and the exponents are listed in Table 2.

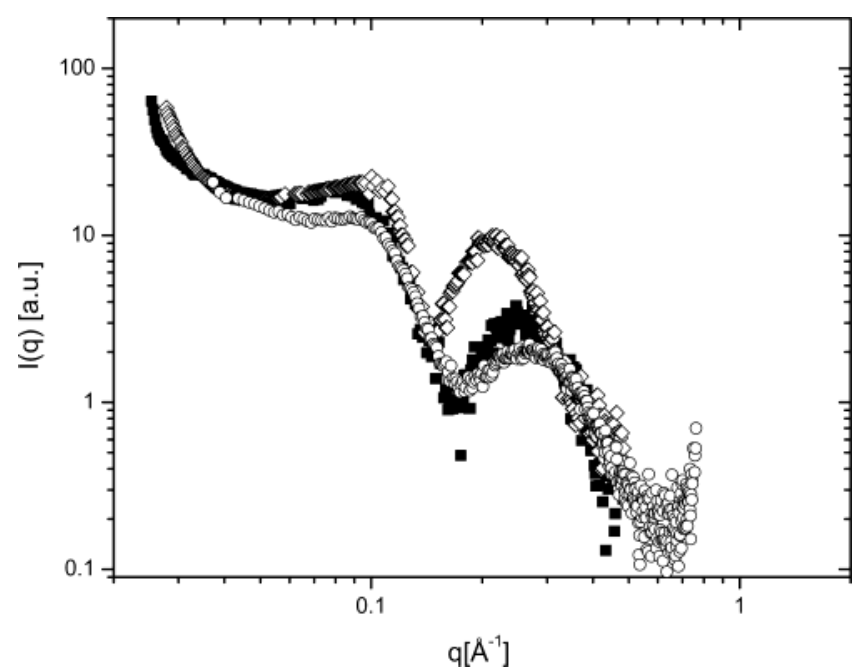

Figure 5. SAXS profiles of $n-C_{8} M A$ with $c_{p}=0.37 \mathrm{~mol} / L$ (open circles), $n-C_{10} M A$ with $c_{p}=0.33 \mathrm{~mol} / L$ (filled squares), and $\mathrm{n}-\mathrm{C}_{14} \mathrm{MA}$ with $\mathrm{C}_{\mathrm{p}}=0.28 \mathrm{~mol} / \mathrm{L}$ (open diamonds).

Table 2. Values of the Exponents for the Mesh Size

Concentration Dependence $\left(\xi \sim \mathrm{c}^{-\alpha}\right)$ from SANS and SAXS Measurements for the Alternating Sodium Maleate Copolymers

\begin{tabular}{llc}
\hline copolymer & \multicolumn{1}{c}{$\alpha$ from SAXS } & $\alpha$ from SANS \\
\hline $\mathrm{n}-\mathrm{C}_{8} \mathrm{MA}$ & $0.55 \pm 0.05(\mathrm{c} \leq 0.29 \mathrm{~mol} / \mathrm{L})$ & $0.37 \pm 0.04$ \\
& $0.15 \pm 0.02(\mathrm{c} \geq 0.29 \mathrm{~mol} / \mathrm{L})$ & \\
$\mathrm{n}-\mathrm{C}_{10} \mathrm{MA}$ & $0.26 \pm 0.03$ & $0.30 \pm 0.03$ \\
$\mathrm{n}-\mathrm{C}_{12} \mathrm{MA}$ & $0.21 \pm 0.06$ & $0.28 \pm 0.03$ \\
$\mathrm{n}-\mathrm{C}_{14} \mathrm{MA}$ & $0.23 \pm 0.03$ & $0.32 \pm 0.03$ \\
DIBMA & $0.5 \pm 0.1$ & $0.43 \pm 0.04$ \\
SMA & $0.5 \pm 0.1$ & $0.45 \pm 0.05$ \\
IBMA & $0.47 \pm 0.05$ &
\end{tabular}

with three different molecular weights. The crossover between unentangled and entangled dynamics is clearly visible.

The inset to Figure 6 shows the specific viscosity for an extended series of increasingly hydrophobic polyelectrolytes which all continue to show the crossover between $c^{1 / 2}$ and $c^{3 / 2}$ scaling, expected by dynamic scaling. The overlap concentration $c^{*}$ of each sample was estimated by extrapolation of the viscosity data to the 


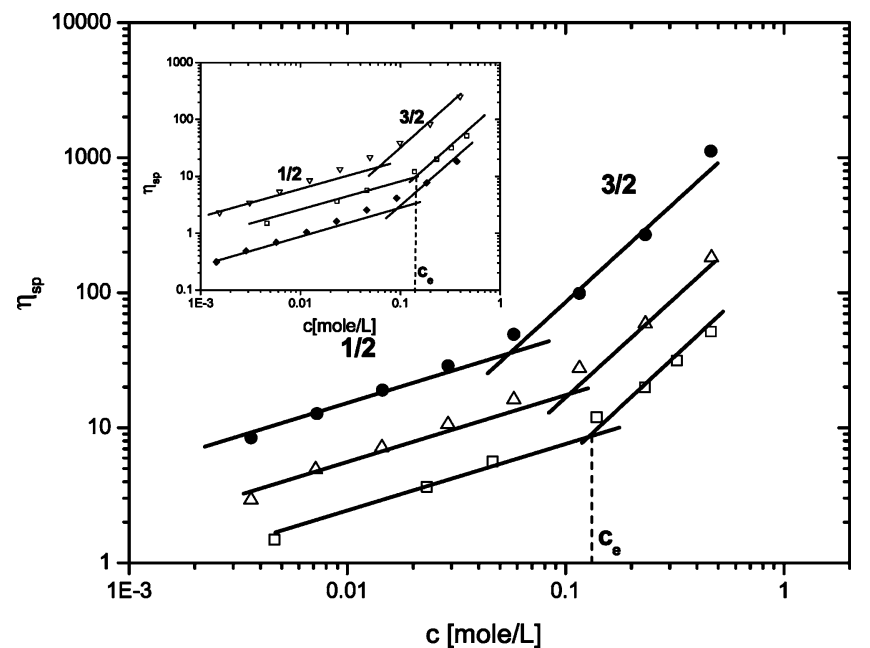

Figure 6. Concentration dependence of the specific viscosity of poly(isobutylene-alt-sodium maleate) (IBMA) of three different molecular weights in water at $25.0^{\circ} \mathrm{C}: \mathrm{M}_{\mathrm{w}}=82000$ $\mathrm{g} / \mathrm{mol}$ (open squares), $M_{w}=196000 \mathrm{~g} / \mathrm{mol}$ (open triangles), $M_{w}$ $=410000 \mathrm{~g} / \mathrm{mol}$ (filled circles). The solid lines are fits to the scaling slopes of $1 / 2$ (unentangled) and $3 / 2$ (entangled). The entanglement concentration $C_{e}$ of the $M_{w}=82000$ sample is shown by the dashed line. Inset: the specific viscosity as a function of copolymer concentration for different low-hydrophobicity copolymers in water at $25.0^{\circ} \mathrm{C}$ : SMA (open down triangles), IBMA (open squares), DIBMA (filled diamonds). The solid lines are fits to the scaling sl opes of $1 / 2$ (unentangled) and $3 / 2$ (entangled). The dashed line indicates the estimated entanglement concentration $C_{e}$ for IBMA with $M_{w}=82000$ $\mathrm{g} / \mathrm{mol}$.

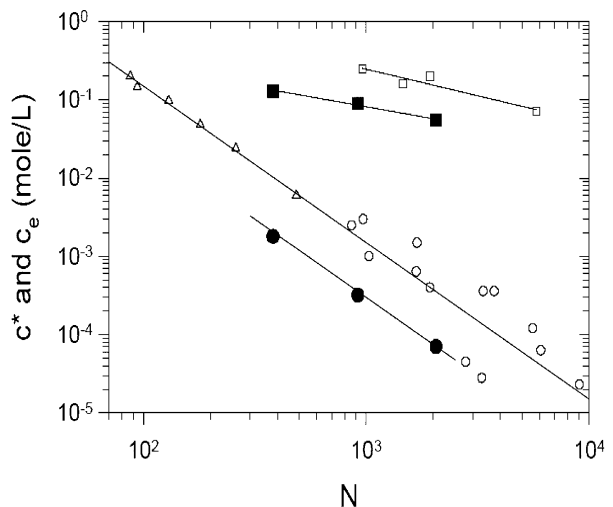

Figure 7. Chain length $(\mathrm{N})$ dependence of the overlap concentration $\mathrm{c}^{*}$ (filled circles) and the entanglement concentration $\mathrm{C}_{\mathrm{e}}$ (filled squares) for three molecular weights of IBMA copolymers in water at $25.0^{\circ} \mathrm{C}$. Data for sodium polystyrenesulfonate ${ }^{24}$ are also shown for comparison (open circles are $c^{*}$ from viscosity, open triangles are $c^{*}$ from SAXS, ${ }^{41}$ and open squares are $c_{e}$ from viscosity $\left.{ }^{24}\right)$. The lines for the overlap concentration have slopes of -2 , as expected from theory, while the lines for the entanglement concentration were determined by linear regression, with slopes of -0.5 for IBMA and -0.7 for NaPSS.

concentration where $\eta_{\mathrm{sp}}=1$, while the crossover between the slopes $1 / 2$ and $3 / 2$ determines the entanglement concentration $\mathrm{C}_{\mathrm{e}}$. The chain length $(\mathrm{N})$ dependences of the overlap concentration and the entanglement concentration for the poly(i sobutylene-alt-sodium maleate) polyelectrolytes are shown in Figure 7.

The measured value of $c^{*} \sim \mathrm{N}^{-2.4 \pm 0.5}$ is in good agreement with the theoretical prediction ${ }^{22}\left(\mathrm{c}^{*} \sim \mathrm{N}^{-2}\right)$. However, the entanglement concentration shows a much weaker chain length dependence $\left(\mathrm{C}_{\mathrm{e}} \sim \mathrm{N}^{-0.6 \pm 0.1}\right)$ than expected by the theory $\left(\mathrm{C}_{\mathrm{e}} \sim \mathrm{C}^{*} \sim \mathrm{N}^{-2}\right)$. Similar behavior

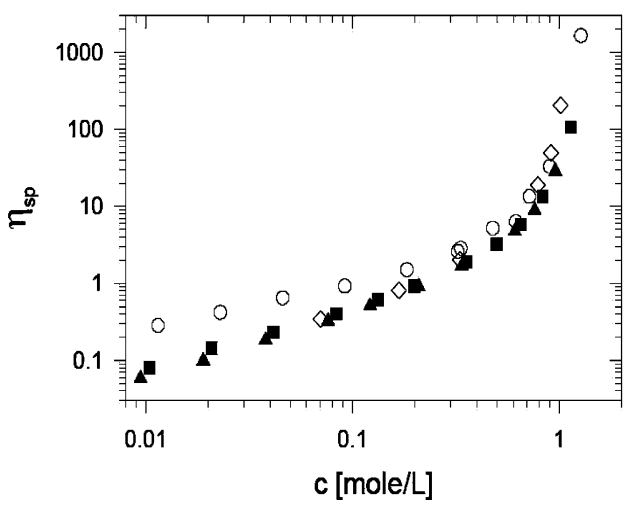

Figure 8. Concentration dependence of the specific viscosity of poly(1-alkene-alt-sodium maleate) copolymers in water at $25.0^{\circ} \mathrm{C}$ : n- $\mathrm{C}_{8} \mathrm{MA}$ (open circles), $\mathrm{n}-\mathrm{C}_{10} \mathrm{MA}$ (filled squares), $\mathrm{n}-\mathrm{C}_{12^{-}}$ MA (filled triangles), $n-C_{14} M A$ (open diamonds).

for the entanglement concentration has previously been observed in the case of poly(styrenesulfonate) (NaPSS), and those data are also shown in Figure 7 for comparison. ${ }^{38}$

For alkene comonomers with more than eight carbons, the scaling now changes from $\eta_{\mathrm{sp}} \sim \mathrm{c}$ bel ow $\mathrm{c}^{*}$ to $\eta_{\mathrm{sp}} \sim$ $c^{4}$ at higher concentrations, as shown in Figure 8. There is thus a very strong dependence of the specific viscosity on polymer concentration for HMPEs above $c^{*}$, in contrast to the case for the less hydrophobic polyelectrolytes.

\section{Discussion}

The samples (Table 1) offer a continuous range of hydrophobicities with identical backbone chemistries. It is important to emphasize that the chain lengths are small (Table 1), discouraging the formation of multimicellar intrapolymer aggregates, which have been pre dicted in the literature. ${ }^{1}$ We divide the polymers into two distinct categories based on chain conformations for dilute solutions: hydrophobic polyelectrolytes ${ }^{22}$ and HMPES. ${ }^{1}$

Hydrophobic Polyelectrolytes. Small-angleX-ray scattering provides better resolution in $\mathrm{q}$ and a wider range of length scales than the corresponding neutron technique, but it is often difficult to quantify the scattering absolutely and neutrons provide different, often advantageous, contrast mechanisms with hydrogencontaining polymers. A combination of the two probes thus provides useful complementary information. From previous studies with X-rays, we deduce that SAXS is dominated by the counterions $\left(\mathrm{Na}^{+}\right){ }^{16}$ With neutrons the hydrogens on the polymer backbone and side chains dominate the signal. ${ }^{39}$

SAXS and SANS show a small angle peak in flexible polyelectrolytes which is due to a correlation hole and not to any periodicity in the system (Figure 1). ${ }^{40}$ The low osmotic compressibility of the counterions pushes down the forward scattering. The peak corresponds to constructive interference of scattering from blobs of charged monomers, and at large $q$ the scattering decays as for polymer chains ( $\mathrm{q}^{-1}$ for rods). Thus, the smallangle scattering is peaked. 40 The HMPEs also have a local maximum that is roughly 1 order of magnitude larger than (and considerably sharper than) the local maximum of the hydrophobic polyel ectrolytes (see Figure 1). The character of this local maximum thereby aids in the separation of these two polymer classes. 
Table 3. Comparison between B Values Calculated from Scattering and Rheology Data;22 Values of B for NaPSS from the Literature ${ }^{24,41}$ Are Also Shown

\begin{tabular}{lccc}
\hline & \multicolumn{3}{c}{ B } \\
\cline { 2 - 4 } copolymer & SAXS & SANS & rheology \\
\hline IBMA04 & 2.7 & & 2.4 \\
IBMA10 & 2.7 & & 2.7 \\
IBMA18 & 2.5 & 2.5 & 2.2 \\
SMA & 3.1 & 2.4 & 3.2 \\
DIBMA & 2.4 & 2.1 & \\
NaPSS & 2.3 & &
\end{tabular}

SAXS/SANS data revealed for all the copolymers with mildly hydrophobic comonomers that the usual polyel ectrolyte behavior 22 is observed, $\xi \sim \mathrm{c}^{-1 / 2}$. The scaling of the mesh size $(\xi)$ as a function of polymer concentration (c) is independent of the molecular weight of the polymer as is clearly shown for the poly(isobutylenealt-sodium maleate) copolymer (Figure 2). This is the expected result for the correlation length in semidilute solution. ${ }^{40}$ Results for the power law fits to eq 3 for all the polymers studied are collected in Table 2.

Dobrynin et al. derive an explicit expression for the scaling of the mesh size of flexible polyelectrolytes in poor solvents. ${ }^{22}$

$$
\mathrm{B}=\xi^{2} \mathrm{cb}
$$

The parameter $B=\mathrm{Nb} / \mathrm{L}$ provides information about the qual ity of the solvent or equivalently the expansion of the chain $(\mathrm{B}>1)$. $\mathrm{N}$ is the number of monomers in the chain, $L$ is the size of the polymer, and $b$ is the monomer length. Thus, the prefactor of the concentration scaling gives additional information about the conformation of the chains. B = 1 corresponds to a fully stretched conformation, and B increases as the chain is less strongly stretched.

The results of fits to eq 4 are collected in Table 3. For the polyelectrolytes made from mildly hydrophobic comonomers the results are close to the literature values for a classic hydrophobic polyelectrolyte system, sodium polystyrenesulfonate, ${ }^{41}$ which has $\mathrm{B}=2.3$.

The analysis of the static structure of the solutions with SAXS/SANS provides a framework in which to understand the concentration dependence of the viscosity. The study of the specific viscosity as a function of concentration shows that the less hydrophobic copolymers behave like typical flexible polyelectrolytes in semidilute solutions, with a change in the scaling from $1 / 2$ to $3 / 2$ as the chains start to entangle (Figure 6). Specifically, we quote the result for the viscosity of the unentangled regime, which provides a separate measure of the extension of the chains $22(B)$.

$$
\mathrm{B}=\mathrm{N}^{2 / 3} \mathrm{c}^{1 / 3} \mathrm{~b} / \eta_{\mathrm{sp}}^{2 / 3}
$$

There is fair agreement between these values and those measured in scattering experiments (Table 3). For comparison, the literature value of B calculated from eq 5 for the sodium salt of polystyrenesulfonate ${ }^{24}$ (NaPSS) is B $=2.8$. The predicted "bead-controlled semi dilute regime" for the hydrophobic polyel ectrolytes ${ }^{38}$ with a unique scaling law, $\eta_{\mathrm{sp}} \sim \mathrm{c}^{0}$, is not observed in Figure 6 or 8.

Experiments of SAXS under shear indicate no change in the form of the correlation peak for low-hydrophobicity sodium maleate copolymers as a function of shear rate in the range $0-500 \mathrm{~s}^{-1}$. The samples studied were
IBMA $04,10,18(3,5 \% \mathrm{w} / \mathrm{w})$, SMA $(3,5 \% \mathrm{w} / \mathrm{w})$, and DIBMA $(3,5 \% \mathrm{w} / \mathrm{w})$. This is expected because these low molar mass polymers show no shear thinning at these rates, in contrast to results on higher molar mass polyel ectrolytes, such as hyaluronic acid and xanthan. ${ }^{42}$

Interestingly, for all the hydrophobic polyelectrolytes studied there is no static signature (X-ray/neutron) of the entangl ement concentration, which is deduced from the rheology (compare Figure 2 and Figure 6). This indicates that the sharp change in chain dynamics is solely due to a dynamic mechanism and not to any particular structural arrangement of the chains relative to one another. Weak aggregation phenomena are observed with DIBMA in dilute solution. ${ }^{31}$ However, the present study indicates the static semidilute solution structure of DIBMA is typical of hydrophobic polyelectrolytes, and it does not share the features of its HMPE counterparts. Apparently, the association observed in dilute solution by light scattering for DIBMA ${ }^{31}$ is not strong enough to perturb the static structure of the semidilute solutions.

Hydrophobically Modified Polyelectrolytes. For the series of $n-C_{m} M A$ copolymers (with $m \geq 8$ ) the scaling of the structure factor peak with concentration reduces from the standard $\sim \mathrm{c}^{-1 / 2}$ scaling of flexible polyel ectrolytes to $\sim \mathrm{C}^{-\alpha}$ with $\alpha<1 / 3$ (Figure 3, Table 2). This behavior is attributed to the interactions of the hydrophobic side chains and water, leading to selfaggregation at low polymer concentrations. The weak concentration dependence suggests that the copolymers $n-C_{m} M A$, made with alkene comonomers with $m>8$ carbons, collapse into gl obular structures. The viscosity data in Figure 8 suggest that these polymers are in dilute solution up to quite high concentrations (of order $0.5 \mathrm{~mol} / \mathrm{L}$, which is comparable to the highest concentrations studied by light scattering). ${ }^{31}$ Interparticle length scaling as $\mathrm{C}^{-1 / 3}$ is the expected result for any dilute solution. A method to calculate the aggregation number ( $\mathrm{N}_{\text {agg }}$ the number of chains per aggregate) is to assume perfect liquid like scaling as done by Waigh et al. 43

$$
N_{\text {agg }}=\frac{C \xi^{3} N_{A v}}{M}
$$

$\mathrm{N}_{\text {Av }}$ is Avogadro's number, and $\mathrm{M}$ is the molar mass of the individual polymers. The average association numbers cal culated using this method are $1.2 \pm 0.3$ chains with $\mathrm{n}-\mathrm{C}_{10} \mathrm{MA}, 2.2 \pm 0.2$ with $\mathrm{n}-\mathrm{C}_{12} \mathrm{MA}$, and $2.5 \pm 0.2$ for $\mathrm{n}-\mathrm{C}_{14} \mathrm{MA}$. This difference in association numbers explains why the correlation lengths do not fall on to a single master curve in Figure 3 . These are slightly smaller than values calculated using Zimm analysis of light scattering in dilute solutions ${ }^{31}\left(\mathrm{~N}_{\text {agg }} \sim 5\right)$ with 0.1 M monovalent salt. Apparently, the stronger electrostatic repulsion with no added salt limits the aggregation. Aggregation numbers calculated from eq 6 as a function of concentration are shown in the inset of Figure 3. $\mathrm{N}_{\text {agg }}$ are seen to increase with concentration as expected from the thermodynamics of these selfassembling materials. ${ }^{44}$ They could also be related to the degree of polydispersity. ${ }^{30}$

The $n-C_{8} M A$ copolymer exhibit a change in the mesh size scaling as the concentration is raised above 0.37 $\mathrm{mol} / \mathrm{L}$; the exponent drops to a value of -0.15 as shown by the SAXS data in the inset of Figure 4. A similar behavior was already observed by Waigh et al., ${ }^{17}$ and it 


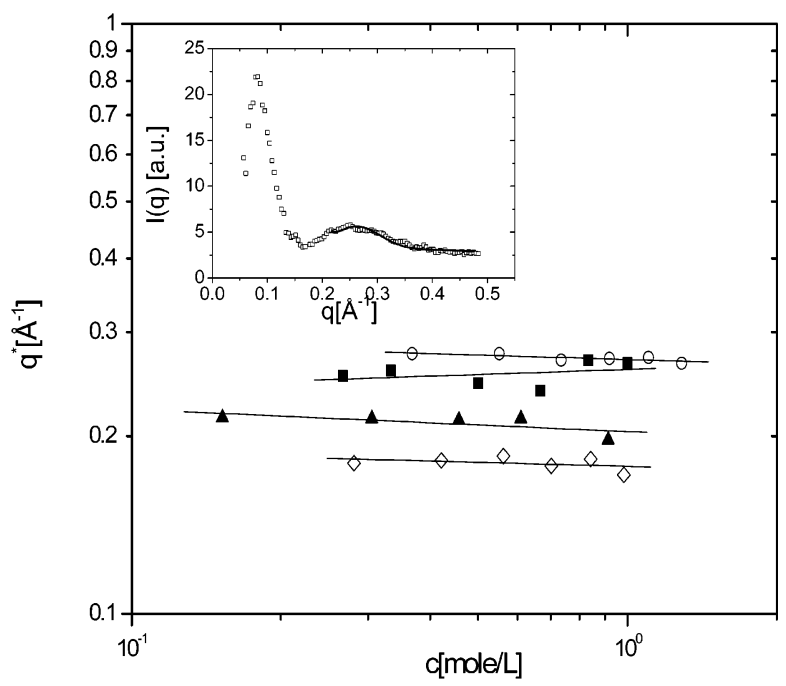

Figure 9. Concentration dependence of the SAXS second peak for the poly(1-alkene-alt-sodium maleate) copolymers: $\mathrm{n}^{-\mathrm{C}_{8}}$ $\mathrm{MA}$ (open circles), $\mathrm{n}-\mathrm{C}_{10} \mathrm{MA}$ (filled squares), $\mathrm{n}-\mathrm{C}_{12} \mathrm{MA}$ (filled triangles), $n-C_{14} \mathrm{MA}$ (open diamonds). The lines are leastsquares fits to power laws. Inset: the polydisperse sphere fit (continuous line) to the small-angle $X$-ray scattering data of $0.33 \mathrm{~mol} / \mathrm{L} \mathrm{n}-\mathrm{C}_{10} \mathrm{MA} .{ }^{49}$ Results from such fits are collected in Table 4.

is attributed to the collapse of highly charged chains in a poor solvent due to self-screening of the electrostatics by the polymer chains (the effective Debye screening length decreases as the polymer concentration increases) and avalanche counterion condensation. ${ }^{45}$ In contrast to the study of Waigh et al., the first scattering peak (Figure 5) is suppressed at yet higher polymer concentrations with $\mathrm{n}-\mathrm{C}_{8} \mathrm{MA}$, but the second peak (spherical form factor) increases in magnitude. This behavior could be due to the onset of steric interactions between the globular chains causing a suppression of the structure factor peak. This has been seen previously for the scattering from micellar systems with soft interparticle potentials. ${ }^{46}$

We relate the wavevector of the second peak in the SAXS data to the size of the compact aggregates, since its position is invariant with concentration (Figure 9). We deduce that the second peak corresponds to invariant morphological features at the nanometer scale. As a first approximation, fits were made with the form factor of a sphere (continuous line inset Figure 9) to the high q data using the assumption of negligible particle interaction at high q on the scattering profiles $(\mathrm{S}(\mathrm{q}) \cong$ 1). ${ }^{37}$ Note that the spherical radii calculated are considerably smaller than those measured in dilute solution (Table 4). This indicates that the chains form intramicellar globules in semidilute solution, since we know that the aggregation numbers are increasing (see inset of Figure 3). The high q tail of $n-C_{8} M A$ was also investigated, as shown in the inset of Figure 10. This ql (q) plot of the SANS data displays a plateau, which is a signature of a rodlike conformation for $n-C_{8} M A$ concentrations bel ow $0.29 \mathrm{~mol} / \mathrm{L}\left(\mathrm{I}(\mathrm{q}) \sim \mathrm{q}^{-1}\right)$. Such a plateau is also observed in Holtzer plots of SANS data from IBMA, SMA, and DIBMA across the complete range of polymer concentrations. Above $0.29 \mathrm{~mol} / \mathrm{L}$ the high $\mathrm{q}$ tail of $\mathrm{n}-\mathrm{C}_{8} \mathrm{MA}$ shows a strong $\mathrm{q}$ dependence, suggesting a structural transition in the system. This might mean that the $n-C_{8}$ MA system exhibits avalanche counterion condensation with the change from a wormlike conformation at low concentrations to a globular
Table 4. Values of the Radius of Gyration from SANS Data of Poly(1-alkene-alt-sodium maleate) Copolymers in the Dilute (Fits of Upturn at Zero q with Spherical Form Factor ${ }^{49}$ ) and Semidilute Regimes (Inset Figure 10, Fits of Oscillation in Scattering to Spherical Form Factor $\left.{ }^{49}\right)^{a}$

\begin{tabular}{cccc}
\hline copolymer & $\begin{array}{c}\mathrm{R}[\mathrm{nm}] \\
\text { (dilute } \\
\text { regime) }\end{array}$ & $\begin{array}{c}\mathrm{R}[\mathrm{nm}] \\
\text { (semidilute } \\
\text { regime) }\end{array}$ & $\begin{array}{c}\mathrm{bN}^{1 / 3}[\mathrm{~nm}] \\
\text { (semidilute } \\
\text { regime) }\end{array}$ \\
\hline $\mathrm{n}-\mathrm{C}_{8} \mathrm{MA}$ & $27 \pm 2$ & $2.30 \pm 0.02$ & 3.1 \\
$\mathrm{n}-\mathrm{C}_{10} \mathrm{MA}$ & $22 \pm 4$ & $2.40 \pm 0.01$ & 2.1 \\
$\mathrm{n}-\mathrm{C}_{12} \mathrm{MA}$ & $21 \pm 2$ & $2.90 \pm 0.01$ & 2.2 \\
$\mathrm{n}-\mathrm{C}_{14} \mathrm{MA}$ & $26 \pm 4$ & $3.50 \pm 0.02$ & 2.2
\end{tabular}

a The experimental values are compared with the theoretical values for a compact globule calculated with $R \cong \mathrm{bN}^{1 / 3}, \mathrm{~b}$ is the monomer size, and $\mathrm{N}$ is the degree of polymerization.

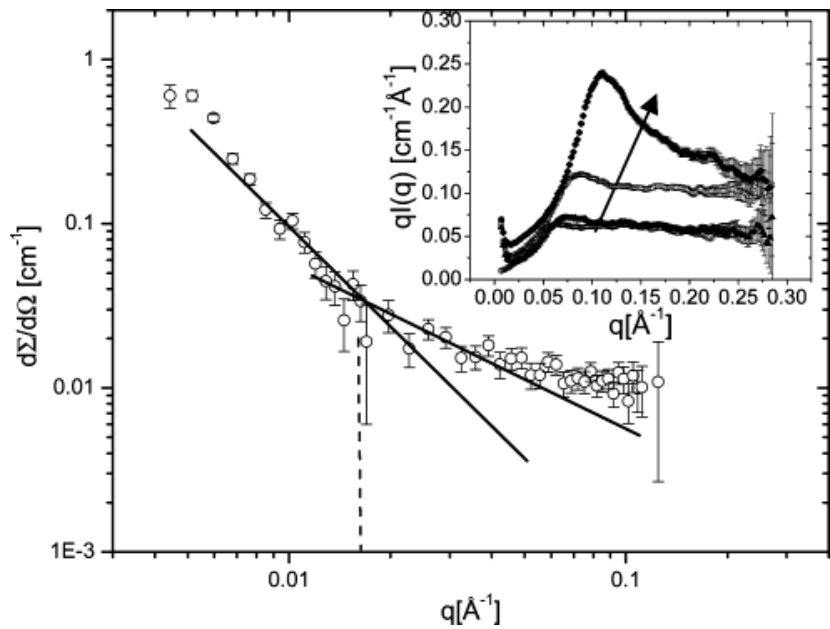

Figure 10. Kratky/Porod crossover from rigid rod $\left(\mathrm{q}^{-1}\right)$ to Gaussian chain scattering $\left(\mathrm{q}^{-2}\right)$ shown by the SANS data for $0.0037 \mathrm{~mol} / \mathrm{L}, \mathrm{n}-\mathrm{C}_{8} \mathrm{MA}$. The intersection at $\mathrm{q}^{*}$ provides an estimate of the persistence length $I_{p}=6 /\left(\pi q^{*}\right)=120 \AA$. Inset: Holtzer plots of the SANS data for the $n-C_{8} M A$ copolymer at four concentrations: $0.092 \mathrm{~mol} / \mathrm{L}$ (open circles), $0.15 \mathrm{~mol} / \mathrm{L}$ (filled triangles), $0.22 \mathrm{~mol} / \mathrm{L}$ (open squares), and $0.37 \mathrm{~mol} / \mathrm{L}$ (filled diamonds). The arrow indicates increasing polymer concentration.

conformation at high concentrations, occurring over a fairly narrow concentration range $(0.29-0.37 \mathrm{~mol} / \mathrm{L}) .45$ The change in morphology coincides with that observed for the mesh size as a function of concentration (Figure 4).

The crossover between $\mathrm{I} \sim \mathrm{q}^{-2}$ and $\mathrm{I} \sim \mathrm{q}^{-1}$ at $\mathrm{q}^{*}$ for $\mathrm{n}-\mathrm{C}_{8} \mathrm{MA}$ indicates that the micelles are cylindrical at low concentrations with a persistence length $I_{p}=6 /\left(\pi q^{*}\right)$ $=120 \AA$ (Figure 10), calculated using the K ratky-Porod model. ${ }^{47}$ Here we are again assuming that $\mathrm{S}(\mathrm{q}) \approx 1$, which is fulfilled in the low concentration regime. Fits to more sophisticated models (e.g., Sharp-Bloomfel d ${ }^{48}$ ) provide similar values for $I_{p}$ with more fit parameters.

We note that the change in forward scattering as a function of polymer concentration was erroneously attributed to a change in the aggregation number in a previous SANS study of alternating lithium maleate copolymers. ${ }^{26}$ Theory points to the osmotic pressure of the counterions dominating the scattering in this regime, for both SAXS and SANS, of polyelectrolyte solutions with no added salt. Thus, we only calculate the radius of gyration from fits of the upturn at low $q$ with a spherical form factor at low concentration of the HMPEs in Table 4. We do not make any statements from this analysis of the upturn in the high concentration regime, where there are the competing effects of 
osmotic pressure and aggregation number. ${ }^{37} \mathrm{~F}$ its were again made for the hydrophobic polymers at dilute concentrations with SANS using the form factor of a polydisperse sphere. ${ }^{49}$

Analysis of the forward scattering (the prefactors of the form factors) indicates that four chains are involved in a single cylindrical aggregate of $n-\mathrm{C}_{8} \mathrm{MA}$ in dilute solution. The chain sizes are collected in Table 4, which allows comparison with values for the semidilute aggregate radii. The first column is from fits of the upturn at zero $q$ in the dilute SANS data, next there is the result from fits, again with a polydisperse spherical form factor $^{49}$ in semidilute solutions, and final ly a theoretical prediction is included using $\mathrm{R} \sim \mathrm{bN}^{1 / 3}$, where $\mathrm{b}$ is the monomer length (5.06 $\AA$ along the backbone direction) and $\mathrm{N}$ is the degree of polymerization. ${ }^{50}$ This theor etical calculation is a lower limit on the size of a complete globular chain since we do not include the contribution of the side chains. Figure 3 (inset) indicates that the aggregation number increases with concentration for $\mathrm{C}_{10} \mathrm{MA}, \mathrm{n}-\mathrm{C}_{12} \mathrm{MA}$, and $\mathrm{n}-\mathrm{C}_{14} \mathrm{MA}$. Thus, we deduce that we are observing connected intrachain micellar globules at the highest concentrations with $n-C_{10} M A, n-C_{12} M A$, and $n-C_{14} M A$, where there is an oscillatory form factor in Figure 5. This necklace of micelles phenomenon has been theoretically predicted for dilute solutions. ${ }^{1}$

We cannot unequivocally differentiate between a proposed cylindrical morphology for $n-C_{8} M A^{26}$ and the string of micelles morphology predicted by Dobrynin and Rubinstein. ${ }^{1}$ E ither scenario expects the $1 /$ q structure factor demonstrated by $n-C_{8} M A$ (Figure 10). Furthermore, both scenarios could account for the smaller aggregate size at high concentrations (Table 4). Wormlike micellar morphologies would be expected to cause a large increase in the viscosity of the polymers which is not observed. The string of micelles structure would provide a smaller density of entangl ements when compared with unassociated polyelectrolytes at the same concentration and thus a lower viscosity. The $n-\mathrm{C}_{8} \mathrm{MA}$ copolymer viscosity is also distinct from the behavior of the copolymers with longer pendant groups, as is shown in Figure 8. The specific viscosities of the copolymers of sodium maleate with $\mathrm{n}-\mathrm{C}_{10}, \mathrm{n}-\mathrm{C}_{12}$, and $\mathrm{n}-\mathrm{C}_{14}$ alkene comonomers fall on the same line, despite the different lengths of the alkyl pendant group. The strong concentration dependence of the viscosity at high polymer concentrations is reminiscent of associating polymer systems as predicted by Rubinstein and Semenov ${ }^{51}$ and observed experimentally with associating polyelectrolytes. 4,6,52 Note in the present study that even with the change in aggregation number as a function of concentration, ${ }^{26}$ the concentration dependence of the specific viscosity of $n-C_{m} M A(m>8)$ is remarkably simple (see Figure 8). The classification of the morphology of each type of copolymer is summarized in Table 1. It is of direct relevance to possible industrial applications that the electrostatic stabilization on a wide range of hydrophobic mesophases can be fine-tuned with the hydrophobicity of the comonomer, moving between polymeric and associating colloidal behavior.

\section{Conclusion}

Both the SANS and SAXS data from sodium mal eate copolymers are consistent with a transition from polyel ectrolyte structure to HMPE structure as the hydrophobicity of the comonomer increases. There is a distinct demarcation between mildly hydrophobic comonomers and hydrophobically modified polyel ectrolyte behavior (observed for alkene comonomers with more than eight carbons). There is a sharp change in the scaling of the mesh size $(\xi)$ with polymer concentration (c) as the size of the pendant groups are increased; from $\xi \sim \mathrm{c}^{-1 / 2}$ for chains with mildly hydrophobic comonomers to $\xi \sim \mathrm{c}^{-\alpha}$ with $\alpha<1 / 3$ for alkene comonomers with more than eight carbons. Similarly, the concentration dependence of viscosity is typical of nonassociating linear polyelectrolytes for chains incorporating the mildly hydrophobic comonomers. There is a crossover between semidilute unentangled $\left(c^{1 / 2}\right)$ and entangled $\left(c^{3 / 2}\right)$ scaling of the specific viscosity as a function of polymer concentration. No structural change is observed with small-angleX-ray and neutron scattering at the entanglement concentrations deduced from rheological measurements, indicating that it is purely a dynamic transition. With the polymers of higher hydrophobicity, associating polymer behavior is observed in semidilute solutions. Specific viscosity is proportional to concentration at low concentrations (below $\mathrm{c}^{*}$ ) but changes to $\eta_{\mathrm{sp}} \sim \mathrm{c}^{4}$ above a critical concentration where the intramolecular globules overlap.

Acknowledgment. We thank Claudine Williams and Michael Rubinstein for useful conversations. We also thank Dr. A. P. Unwin for hel ping with our in-house X-ray facility and Nick Terril for assistance at the Daresbury synchrotron.

\section{References and Notes}

(1) Dobrynin, A. V.; Rubinstein, M. Macromolecules 2000, 33, 8097.

(2) Semenov, A. N.; Rubinstein, M. Macromolecules 1998, 31, 1373.

(3) Kotz, J .; Kosmella, S.; Beitz, T. Prog. Polym. Sci. 2001, 26, 1199.

(4) Bromberg, L. Macromolecules 1998, 31, 6148.

(5) Guenoun, P.; Davis, H. T.; Tirrell, M.; Mays, J. W. Macromolecules 1996, 29, 3965.

(6) Wientjes, R. H. W.; Duits, M. H. G.; J ongschaap, R. J . J .; Mellema, J. Macromol ecules 2000, 33, 9594.

(7) Kujawa, P.; Rosiak, J . M.; Selb, J .; Candaua, F. Macromol. Chem. Phys. 2001, 202, 1384.

(8) Dobrynin, A. V.; Rubinstein, M. Macromolecules 1999, 32, 915.

(9) Laschewsky, A. Adv. Polym. Sci. 1995, 124, 1.

(10) Braun, O.; Boue, F.; Candau, F. Eur. Phys. J . E 2002, 7, 141.

(11) Dobrynin, A. V.; Rubinstein, M.; Obukhov, S. O. Macromolecules 1996, 29, 2974

(12) Micka, U.; Holm, C.; Kremer, K. Langmuir 1999, 15, 4033.

(13) Baigl, D.; Ober, R.; Qu, D.; Fery, A.; Williams, C. E. Europhys. Lett. 2003, 62, 588 .

(14) Heitz, C.; Rawiso, M.; Francois, J. Polymer 1999, 40, 1637.

(15) Aseyev, V. O.; Klenin, S. I.; Tenha, H.; Grillo, I.; Geissler, E. Macromol ecules 2001, 34, 3706.

(16) Essafi, W.; Lafuma, F.; Williams, C. E. J . Phys. II 1995, 5, 1269.

(17) Waigh, T. A.; Ober, R.; Williams, C. E.; Galin, J . C. Macromolecules 2001, 34, 1973.

(18) Kiriy, A.; Gorodyska, G.; Minko, S.; J aeger, W.; Stepanek, P.; Stamm, M. J. Am. Chem. Soc. 2002, 124, 13454.

(19) Minko, S.; Kiriy, A.; Gorodyska, G.; Stamm, M. J . Am. Chem. Soc. 2002, 124, 3218.

(20) Serwer, P.; Hayes, S. J . Biophys. J . 2001, 81, 3398.

(21) Borisov, O. V.; Halperin, A. Macromolecules 1996, 29, 2612.

(22) Dobrynin, A. V.; Colby, R. H.; Rubinstein, M. Macromolecules 1995, 28, 1859.

(23) Oostwal, M.; Odijk, T. Macromolecules 1993, 26, 6489.

(24) Boris, D. C.; Colby, R. H. Macromolecules 1998, 31, 5746.

(25) Tirtaatmadja, V.; Tam, K. C.; J enkins, R. D. Macromolecules 1997, 30, 3271.

(26) Shih, L. B.; Sheu, E. Y.; Chen, S. H. Macromolecules 1988, 21, 1387. 
(27) Shih, L. B.; Mauer, D. H.; Verbrugge, C.J .; Wu, C. F.; Chang, S. L.; Chen, S. H. Macromol ecules 1988, 21, 3235.

(28) Chitanu, G. C.; Skouri, M.; Schosseler, F.; Munch, J. P.; Carpov, A.; Candaua, S. J . Polymer 2000, 41, 3683.

(29) di Cola, E.; Waigh, T. A.; Colby, R. H.; Tan, J ., manuscript in preparation.

(30) Sauvage, E.; Amos, D. A.; Antalek, B.; Schroeder, K. M.; Tan, J. S.; Plucktaveesak, N.; Colby, R. H. Submitted toJ . Polym. Sci., Polym. Phys. Ed.

(31) Sauvage, E.; Plucktaveesak, N.; Colby, R. H.; Amos, D. A.; Antalek, B.; Schroeder, K. M.; Tan, J. Submitted to J . Polym. Sci., Polym. Phys. Ed.

(32) LOQ. http://www.isis.rl.ac.uk.

(33) Heenan, R. K.; Penfold, J .; King, S. M. J . Appl. Crystallogr. 1997, 30, 1140.

(34) Wignall, G. D.; Bates, F. S. J . J . Appl. Crystallogr. 1987, 20 28.

(35) Bras, W.; Derbyshire, G. E.; Ryan, A. J .; Mant, G. R.; Murry, P. K.; Roberts, K.; Sumner, I.; Warges, J. S.; Lewis, R. A.; Gabriel, A. Rev. Sci. Instrum. 1989, 60, 2346.

(36) http://www.srs.ac.uk/srs/beamlines.html.

(37) Higgins, J. S.; Benoit, H. C. Polymers and Neutron Scattering; OUP: New York, 1994.

(38) Plucktaveesak, N. Pennsylvania State, 2003.

(39) Nierlich, M.; Williams, C. E.; Boue, F.; Cotton, J . P.; Daoud, M.; Farnoux, B.; J annick, G.; Picot, C.; Moan, M.; Wolf, C.; Rinaudo, M.; de Gennes, P. G.J . Phys. (Paris) 1979, 40, 701.
(40) de Gennes, P. G.; Pincus, P.; Velasco, R. M.; Brochard, F. J . J. Phys. (Paris) 1976, 37, 1461.

(41) Kaji, K.; Urakawa, T.; Kanaya, T.; Kitamaru, R. J . Phys. (Paris) 1988, 49, 993.

(42) Villetti, M.; Borsali, R.; Diat, O.; Soldi, V.; Fukada, K. Macromol ecules 2000, 33, 9418.

(43) Waigh, T. A.; Papagiannopoulos, A.; Voice, A.; Bansil, R.; Unwin, A. P.; Dewhurst, C.; Turner, B.; Afdhal, N. Langmuir 2002, 18, 7188.

(44) Evans, D. F.; Wennerstron, H. The Colloidal Domain; Wiley: New York, 1994

(45) Dobrynin, A. V.; Rubinstein, M. Macromolecules 2001, 34, 1964.

(46) Watzlawek, M.; Lowen, H.; Likos, C. N. J . Phys.: Condens. Matter 1998, 10, 8189.

(47) Kratky, O. In Small-Angle X-ray Scattering; Glatter, O., Kratky, O., Eds.; Academic Press: New York, 1982.

(48) Sharp, P.; Bloomfield, V. A. Biopolymers 1968, 6, 1201.

(49) Kotlarchyk, M. J . Chem. Phys. 1983, 79, 2461.

(50) Grosberg, A. Y.; Khokhlov, A. R. Statistical Physics of Macromolecules; AIP Press: Woodbury, NY, 1994.

(51) Semenov, A. N.; Rubinstein, M. Macromolecules 1998, 31, 1373.

(52) Regalado, E. J .; Selb, J .; Candau, F. Macromolecules 1999, 32,8580 .

$\mathrm{MA049260 \textrm {H }}$ 